Linguistique, littérature, didactique

\title{
Les didactiques et la question des littéracies universitaires
}

Yves Reuter

\section{OpenEdition}

\section{Journals}

Édition électronique

URL : http://journals.openedition.org/pratiques/1979

DOI : 10.4000/pratiques. 1979

ISSN : 2425-2042

Éditeur

Centre de recherche sur les médiations (CREM)

Édition imprimée

Date de publication : 15 juin 2012

Pagination : 161-176

Référence électronique

Yves Reuter, "Les didactiques et la question des littéracies universitaires », Pratiques [En ligne], 153-154 | 2012, mis en ligne le 13 juin 2014, consulté le 20 avril 2019. URL : http:// journals.openedition.org/pratiques/1979; DOI : 10.4000/pratiques.1979

(c) Tous droits réservés 


\title{
Les didactiques et la question des littéracies universitaires
}

\author{
Yves Reuter
}

Université de Lille 3

Laboratoire Théodile-CIREL (E.A. 4354)

J'aborderai dans cet article ${ }^{(1)}$ les quatre questions suivantes ${ }^{(2)}$. En premier lieu, les déplacements dans les manières de penser les contenus et les écrits à l'université, ainsi que les difficultés qui sont attachées à ces écrits et les remédiations possibles, qu'implique le simple fait d'avancer le syntagme de littéracies universitaires. Puis j'interrogerai les disciplines de recherche qui ont plus ou moins traité ces questions, ou qui sont susceptibles de le faire, en essayant de préciser quelles limites présente, à mon sens, leur mode d'appréhension de ces problèmes. Je m'attacherai ensuite à spécifier les singularités et les intérêts de la perspective propre aux didactiques, en tant que disciplines de recherche, pour penser ces questions avant de conclure sur les problèmes que l'approche didactique rencontre et qu'elle doit tenter de résoudre.

\section{Six déplacements d'ampleur}

Il me parait important, en premier lieu, de prendre la mesure des déplacements dans les manières classiques de penser l'écrit, les contenus, la formation et la recherche - au moins en France - qu'induit le simple fait de poser l'expression littéracies universitaires comme titre d'une recherche et d'un colloque ré-

(1) Cet article est une réécriture de ma communication au colloque international : Littéracies universitaires : savoirs, écrits, disciplines, organisé à l'Université de Lille 3, les 2, 3 et 4 septembre 2010 (I. Delcambre, D. Lahanier-Reuter, F. Boch, dir.).

(2) Je ne développerai pas ici une approche historique des questions posées, maitrisant insuffisamment l'histoire des littéracies universitaires et des problèmes qu'elles ont suscités à l'échelon international. Il me semble cependant qu'il s'agit là d'un projet de recherche comparatiste particulièrement stimulant. 
unissant des chercheurs issus de diverses communautés de recherche ${ }^{(3)}$. Je m'attarderai plus particulièrement sur six de ces déplacements que je considère majeurs.

\subsection{Des techniques aux pratiques sociales}

Le premier d'entre eux me parait résider en ce que la lecture et l'écriture ne sont plus considérées, comme c'est encore majoritairement le cas à tous les niveaux de l'institution scolaire, comme des techniques indépendantes de leur conditions d'exercice mais comme des pratiques sociales ${ }^{(4)}$, tributaires à ce titre des contenus, des situations, des enjeux, des communautés, des sphères socio-institutionnelles... Complémentairement, langue ou écrit, ne sont pas considérés essentiellement comme des codes, comme des ensembles d'unités et de règles d'assemblage de ces unités, comme des objets dotés d'une existence (relativement) indépendante mais sont appréhendés sous l'angle de leurs conditions de possibilité, de leurs usages et de leurs effets par et pour des sujets donnés dans des contextes sociaux déterminés. L'arrière-plan convoqué me parait ainsi se déplacer de modélisations issues de la tradition scolaire, voire de certaines approches théoriques de la langue (sensiblement édulcorées par rapport à leur complexité originelle) à des cadres sollicitant plutôt l'héritage bakhtinien, la socio-linguistique, les approches socio- et ethno-logiques de l'écrit ${ }^{(5)}$, les travaux sur le rapport à l'écrit ${ }^{(6)}$ ou sur le langage au travail ${ }^{(7)} \ldots$

\subsection{Des savoirs « abstraits» aux pratiques (discursives) des savoirs}

Le second déplacement, indissociable du premier et aux conséquences tout aussi remarquables, me parait porter sur les conceptions mêmes des savoirs qui n'ont plus, dans ce cadre, une existence « abstraite », en quelque sorte indépendante de leurs formes langagières et des pratiques de production et de réception qui les mettent en œuvre. Les savoirs sont ainsi considérés comme formatés par des genres, des écrits, des pratiques de lecture et d'écriture... qui les instituent socialement et qui font qu'un sujet social n'est jamais confronté à un savoir purement "idéel » mais à un savoir institué langagièrement, cette institution structurant, de manière déterminante, qu'elle soit facilitante ou non, l'appropriation $\mathrm{du}$ « contenu de savoir».

\subsection{De fonctionnements universitaires peu interrogés à la perception d'un échec inadmissible}

Le troisième déplacement induit par le fait de poser l'expression littéracies

(3) Sur l'absence d'évidence de l'introduction du terme de littéracies dans le champ des didactiques, voir par exemple Lidil, 2003, et Barré de Miniac, Brissaud, Rispail, dir., 2004.

(4) Sur cette notion, voir Reuter, 1996, pp. 58-65. J'y reviendrai dans la suite cet article.

(5) Voir, par exemple, les travaux de Goody (notamment Goody, 1977).

(6) Voir, entre autres, Lahire, 1993 ou Charlot, Bautier, Rochex 1992.

(7) Par exemple, Borzeix et Fraenckel, 2001. 
universitaires me parait résider dans une prise de conscience qui entraine une analyse critique des fonctionnements universitaires classiques dans la mesure où :

- ils n'ont rien de naturel, ainsi qu'en attestent les études sur leurs variations diachroniques et synchroniques;

- ils engendrent, ou au minimum n'empêchent pas, des difficultés voire un échec important chez les étudiants ;

- cet échec est socialement préoccupant ;

- cet échec ne peut être attribué « simplement » aux capacités cognitives des étudiants (qui seraient considérées a priori comme insuffisantes).

\subsection{D'un constat fataliste à un projet d'action légitime}

Mais le constat de cet échec, aussi préoccupant soit-il, me parait encore être accompagné par un quatrième déplacement, loin d'avoir été évident ou, en tout cas, loin d'être mécaniquement lié au précédent. Il consiste à penser que les difficultés rencontrées sont légitimement traitables à l'université, i.e. que leur traitement ne disqualifie ni l'université, ni les universitaires qui s'en chargent, sous prétexte qu'il « secondariserait », voire qu'il « primariserait » l'université et de surcroit qu'elles sont possiblement traitables à l'université. Ce qui signifie que tout ne se joue pas avant cinq ans... ou avant dix-huit ans. Ce qui signifie encore un changement de posture consistant à ne plus rejeter les problèmes sur les étapes antérieures du cursus pour mieux éviter de les traiter ou d'avouer son impuissance à les traiter, comme c'est encore trop souvent le cas, au moins en France

\subsection{De la répétition à des dispositifs de remédiation adossés aux pratiques universitaires}

Le cinquième déplacement, même s'il peut sembler structurellement attaché au précédent, n'a cependant, lui non plus, rien d'évident, référé aux fonctionnements universitaires classiques. Il consiste à poser que les interventions envisageables (et potentiellement efficaces) ne portent pas principalement sur la maîtrise de la langue (par exemple la syntaxe ou l'orthographe) « en général» ou sur des savoir-faire (la lecture et l'écriture considérées comme des techniques « autonomes »), c'est-à-dire sur ce qui a été étudié avant l'entrée à l'université et dans des formes identiques à celles de la scolarité antérieure, mais que ces interventions possibles reposent prioritairement sur l'analyse des pratiques disciplinaires propres aux études universitaires (et cela, aux différentes étape du cursus et selon les différentes filières), sur l'analyse des représentations associées à ces pratiques (que ce soit celles des étudiants ou celles des enseignants), sur l'analyse des dispositifs d'enseignement eux-mêmes en tant qu'ils s'avèrent insuffisamment efficaces, voire qu'ils sont susceptibles d'engendrer certaines de ces difficultés... 


\subsection{De la disqualification à la légitimité des littéracies universitaires comme objets de recherche possibles}

Le dernier déplacement que j'évoquerai ici est peut-être le plus étonnant au regard des traditions universitaires et de la légitimité des objets de recherche ${ }^{(8)}$. Il consiste à poser que travailler en recherche sur l'échec des étudiants et sur les manières d'y remédier, sur la lecture et l'écriture à l'université, sur les difficultés qui leur sont liées, sur les stratégies d'intervention possibles et sur leurs effets sont des objets de recherche tout aussi nobles que les blancs chronologiques chez Flaubert, la théorie du chaos, les neutrinos ou la pensée de Heidegger...

\section{Quelques limites des disciplines de recherche traitant de ces problèmes}

Après avoir précisé les déplacements en jeu, il convient maintenant de s'interroger sur les disciplines de recherche traitant - ou susceptibles de traiter ces questions et sur leurs limites, limites qui justifient à mes yeux l'approche didactique que je présenterai immédiatement après.

\subsection{Une réelle multiplicité de disciplines...}

De multiples disciplines de recherche ont montré qu'elles pouvaient apporter des éclairages importants sur les questions qui nous préoccupent ici. Je citerai ainsi, à titre d'exemples et sans volonté aucune d'exhaustivité ${ }^{(9)}$, l'histoire de l'éducation, lorsqu'elle étudie, dans la perspective diachronique qui est la sienne, les modes d'enseignement et les modalités de travail des étudiants à l'université, la sociologie dans ses composantes qui se préoccupent des pratiques culturelles, des pratiques de lecture et d'écriture des étudiants à l'université ou de l'échec en tant qu'il est socialement différencié ${ }^{(10)}$, l'anthropologie lorsqu'elle analyse les différences entre ordre de l'écrit et ordre de l'oral, la psychologie (par exemple cognitive) lorsqu'elle tente de préciser certaines opérations mentales qui seraient impliquées dans la lecture ou l'écriture, ou encore les sciences du langage et des textes avec, entre autres, les travaux sur les modes de structuration de l'écrit ou sur les stratégies d'écriture (voir, par exemple, dans ces dernières décennies, les apports de la génétique textuelle)... Il serait donc absurde de se priver des contributions indéniables de ces disciplines.

(8) Sur la question de la légitimité des objets de recherche, voir Bourdieu, 1984 ou Bourdieu, Chartier, 2010.

(9) Je prie le lecteur de bien vouloir m'excuser pour la suspension des références dans le passage suivant : les éléments bibliographiques à mentionner seraient, en l'occurrence, pléthoriques.

(10) On conviendra que, sur ce terrain, les chercheurs ont une dette important à l'égard de sociologues tels que Bourdieu et Passeron. 


\section{2. ...mais des limites partagées}

Cependant toutes ces disciplines de recherche présentent, à mon sens, des limites non négligeables pour appréhender les questions soulevées ici. Je ne mentionnerai ici que deux d'entre elles qui me paraissent particulièrement importantes.

La première limite tient à des problèmes d'échelle. Ou, pour le dire sous une autre forme, le prisme au travers duquel ces disciplines s'emparent de ces questions me parait bien souvent soit trop large (par exemple les différences entre écrit et oral à l'échelle des sociétés ou encore l'écriture « en général (i.e. indépendamment de son inscription dans telle ou telle sphère socio-institutionnelle et de son formatage par des pratiques et des genres déterminés...), soit trop restreint (par exemple la dimension orthographique des écrits ou encore les dimensions cognitives de l'écriture, envisagées indépendamment de leurs relations avec les autres composantes des écrits en jeu...).

La seconde limite tient aux dissociations fréquemment effectuées par nombre de ces travaux, d'une part entre contenus et pratiques langagières (cela reste, de fait, le cas d'une majorité de disciplines qui ont tendance à mésestimer le poids de la part langagière dans la structuration des savoirs ${ }^{(11)}$ ), d'autre part entre contenus ou disciplines et enseignement et apprentissages (comme si, à l'université, les disciplines et les contenus existaient indépendamment des pratiques d'enseignement et d'apprentissages ${ }^{(12)}$ qui les constituent).

J'ajouterais volontiers que, par voie de conséquence, les formalisations proposées s'avèrent, à de rares exceptions près, peu pertinentes pour éclairer les difficultés des apprenants.

\subsection{Un agglomérat d'éclairages non articulés}

Cette situation - apports indéniables mais aussi limites de nombre de disciplines - me parait aboutir à une juxtaposition d'éclairages, possiblement complémentaires mais de fait disparates, en tout cas non articulés, ainsi qu'en témoignent les colloques monodisciplinaires sur ces questions, colloques au sein desquels les spécialistes d'une discipline donnée débattent entre eux dans l'ignorance des recherches menées dans les autres disciplines, ou encore les colloques pluridisciplinaires où les chercheurs des différentes disciplines interviennent encore trop souvent en méconnaissant tout autant les apports de leurs collègues d'autres disciplines (ainsi que le manifestent très clairement les citations et les références bibliographiques utilisées )...tout en regrettant que ceux-ci ne lisent pas plus leurs travaux.

(11) Et cela d'autant plus qu'on avance dans le cursus, comme si cette «avancée » dans le cursus s'accompagnait d'une plus grande pureté conceptuelle...

(12) D'une certaine manière, il s'agirait de ne pas de confondre ces " maitres en disciplines » que sont les universitaires qui se baptisent d'ailleurs linguistes, philosophes, physiciens ou sociologues... avec de simples enseignants, voire avec des maitres d'école... 


\section{Spécificités et intérêts de la perspective didactique}

Il me semble que, dans le cadre ainsi tracé, les didactiques permettent quelques déplacements importants, points d'appuis possibles pour des avancées non négligeables.

\subsection{Deux différences majeures}

Je rappellerai d'abord, afin d'éviter tout malentendu aussi bien dans l'espace francophone que dans l'espace anglo-saxon, la définition des didactiques que j’avais proposée :

«[...] Les didactiques [sont] les disciplines de recherche qui analysent les contenus (savoirs, savoir faire...) en tant qu'ils sont objets d'enseignement et d'apprentissages, référés/référables à des matières scolaires »(Reuter, 2007b, p. 69)

Ainsi défini, le projet de connaissance des didactiques implique deux différences majeures par rapport aux autres disciplines de recherche évoquées. En premier lieu, pour les didactiques, l'articulation entre contenus, enseignement et apprentissages est fondamentale qu'il s'agisse des relations entre contenus " savants " (i.e. institutionnellement désignés comme savants) et contenus à enseigner (i.e. institutionnellement désignés comme étant à enseigner), ou des relations entre contenus à enseigner et contenus réellement/pratiquement enseignés, ou encore des relations entre contenus enseignés et contenus appris...

En second lieu, pour les didactiques, l'articulation entre contenus (savoirs, savoir-faire, valeurs, rapports à, manières de penser, d'agir, de discourir...) et pratiques langagières est tout aussi fondamentale ainsi qu'en attestent divers concepts parmi les plus utilisés lors de ces dernières décennies dans le champ des didactiques. Je n'en prendrai ici, et trop succinctement sans doute, que trois exemples.

Ainsi, le concept de texte du savoir, proposé par Yves Chevallard (1985/1991), à partir du champ de la didactique des mathématiques, éclaire les modalités selon lesquelles les savoirs «savants », pour être enseignables et enseignés, doivent être mis en texte de manière spécifique, via par exemple programmes ou manuels scolaires ${ }^{(13)}$. Le concept de niveau de formulation, proposé par Jean-Pierre Astolfi et Michel Develay, sur le terrain de la didactique des sciences ${ }^{(14)}$, éclaire complémentairement le fait que la formulation des savoirs en jeu dans une situation d'enseignement et d'apprentissages varie selon le moment du cursus et les problèmes spécifiques étudiés. A un autre niveau, le concept de forme scolaire, proposé par le sociologue Guy Vincent (1980, dir. 1994), retravaillé par Bernard Lahire (2008), et repris par divers didacticiens, notamment du français, associe étroitement fonctionnements scolaires et ordre

(13) Sur ce point, voir aussi Daunay, 2007.

(14) Voir Astolfi et Develay, 1989 ; Astolfi et alii ,1997 et, pour une synthèse récente CohenAzria, 2007. 
de l'écrit en montrant comment, dans nos cultures, l'école s'appuie sur l'ordre de l'écrit (plus que sur celui de l'oral), via organisation et codification des savoirs et des savoir-faire, pratiques et attitudes sollicitées. À ces concepts, il serait sans doute nécessaire d'associer encore ceux de genre ou de métalangage (Delcambre, 2007a et b) aux usages tout aussi répandus et stratégiques au sein des didactiques.

En outre, cette articulation fondamentale entre contenus et pratiques langagières n'est pas uniquement attestée par l'appareil conceptuel des didactiques ; elle repose également sur les méthodes et les documents de recherche privilégiés par les didacticiens, notamment l'analyse des textes prescriptifs (programmes...), des manuels scolaires, des consignes des exercices, des productions des élèves, des annotations des enseignants...

\subsection{De quelques intérêts spécifiques liées à ces différences}

Il me semble, par voie de conséquence, que le projet de connaissance des didactiques, avec les deux différences majeures par rapport aux autres disciplines de recherche que je viens d'évoquer, entraine quelques intérêts spécifiques quant aux questions en jeu. Je n'en retiendrai ici que cinq parmi ceux qui me paraissent les plus déterminants.

Le premier de ces intérêts consiste en la dénaturalisation de la notion de discipline, dénaturalisation essentielle en ce qu'elle peut permettre de penser les cadres au travers desquels chacun d'entre nous pense sans avoir véritablement conscience de la manière dont ces cadres formatent ses manières de penser. Dans cette perspective, nous avons été amenés - au sein de notre laboratoire (Theodile-CIREL) - à distinguer soigneusement disciplines scolaires, disciplines universitaires et disciplines de recherche, ce qui permet, à mon sens, d'éviter certaines confusions entre savoirs ou savoir-faire institutionnellement retenus dans des filières de formation et savoirs ou savoir-faire en train d'être élaborés dans les espaces de recherche, ce qui permet aussi de se donner les moyens d'étudier les interactions entre ces trois espaces disciplinaires, ce qui permet encore d'appréhender - à la différence de certains discours conservateurs et/ou élitistes - les disciplines universitaires comme des disciplines d'enseignement et d'apprentissages. Toujours dans cette même perspective de dénaturalisation de la notion de discipline, nous avons été amenés à proposer le concept de configuration disciplinaire (Reuter, Lahanier-Reuter, 2007a) qui permet de différencier les diverses modalités d'actualisation des disciplines selon les moments du cursus, les filières, les pays, les périodes historiques, les espaces sociaux (discipline prescrite / recommandée / représentée / pratiquée... ${ }^{(15)}$. Cela permet ainsi d'éviter les visions essentialistes et monolithiques (empiriquement peu fondées) des disciplines et incite à étudier plus précisément les relations et les interactions (ainsi que leurs causes et effets possibles) entre ces diverses formes d'actualisation des disciplines.

(15) Pour plus de précisions sur ces distinctions entre espaces, voir Reuter, 2007a. 
Le second intérêt, lié au projet de connaissance des didactiques, consiste à mon sens en l'élaboration de concepts susceptibles de contribuer à préciser les fonctionnements des littéracies universitaires. Ici encore, faute d'espace, je n'en évoquerai que deux (étroitement articulés d'ailleurs avec le concept de configuration disciplinaire que je viens d'évoquer). En premier lieu, le concept de conscience disciplinaire (Reuter, 2003 et 2007c) peut être défini comme la manière dont les apprenants (ou d'autres acteurs) (re)construisent les disciplines. Il permet de préciser les « images » de la discipline, les manières dont les institutions ou les acteurs se les représentent et, par voie de conséquence, certains conflits ou malentendus possibles entre enseignants et apprenants. Ainsi en est-il, par exemple, lorsque les apprenants se représentent la littérature comme une discipline où l'important est d'exprimer ses émotions et/ou de «baratiner » ou les mathématiques comme une discipline où les formes langagières sont secondaires (Lahanier-Reuter, Reuter, 2002). Le second concept, celui d'univers de l'écrit que j'ai proposé avec Jacinthe Giguère (Giguère, Reuter, 2004 ; Reuter, Lahanier-Reuter, 2007b), s'appuie, dans le cadre d'une approche matérialiste ${ }^{(16)}$ des phénomènes scolaires et universitaires, sur trois principes complémentaires :

- les activités des apprenants - en l'occurrence la lecture et l'écriture - gagnent à être analysées en tant qu'elles sont actualisées dans des pratiques $^{(17)}$;

- ces pratiques sont modélisantes dans la mesure où elles donnent forme et structurent le sens des activités, des représentations des acteurs, des relations établies entre écrits et savoirs ainsi que nombre d'effets (appropriation, investissement, résistance...);

- ces pratiques sont elles-mêmes structurées par des univers - de l'écrit/de l'enseignement de l'écrit - entendus comme des systèmes, articulant dans un espace socio-institutionnel déterminé (ici l'université), les composantes de l'écrit, tout en organisant leur fonctionnement dans un ensemble de situations ${ }^{(18)}$.

Le troisième intérêt lié au projet de connaissance des didactiques tient à ce qu'elles peuvent, en fonction de ce projet et de leurs principes constitutifs, prendre appui sur l'analyse des difficultés et des représentations des apprenants pour penser les pratiques et construire des modélisations des écrits, non

(16) Ici encore fortement redevable aux travaux de Pierre Bourdieu.

(17) Sur cette notion, voir la note 4. Il s'agit en fait de ne pas extraire de l'analyse de ce qui est désigné comme «activités » les modalités physiques de leur exercice, l'articulation entre les dimensions cognitives, émotionnelles et sociales, leur insertion dans l'ensemble de la vie des sujets, leur histoire, leurs modes de relation avec les autres activités...

(18) Afin de spécifier ces univers de l'écrit, nous avons construit une grille d'analyse organisée autour de six dimensions : les catégories d'écrits ; les supports ; les outils ; les pratiques ; les articulations entre codes - écrit/oral -, entre systèmes sémiotiques (verbal et iconique par exemple) entre espaces - scolaire et extrascolaire - ; les normes et leurs modalités de fonctionnement. 
indépendantes des pratiques et des modalités d'apprentissage des écrits et des pratiques qui les constituent. Ainsi, Bernard Delforce, par exemple, a pu montrer dans cette perspective, de manière convaincante, notamment à partir d'analyses de dissertations et de réactions d'élèves face aux corrections des enseignants, que les représentations des apprenants constituaient des systèmes généralement cohérents (tout à la fois facteurs d'aide et obstacles possibles dans la réalisation des objets langagiers), éclairant de manière intéressante le fonctionnement des pratiques. Il a aussi parfaitement montré, à mon sens, comment les difficultés rencontrées par les apprenants renvoyaient souvent à des lieux problématiques des écrits eux-mêmes incitant ainsi les chercheurs à préciser la structuration de ces écrits et la manière de gérer les problèmes que celle-ci engendre $^{(19)}$ (Delforce, 1985 et 1986). Ainsi construite l'analyse des difficultés des apprenants n'est donc nullement l'application de thèses construites a priori, en dehors des discours et des réalisations des apprenants. Elle n'est pas simplement inscrite dans une logique « descendante » où le terrain de l'enseignement et des apprentissages est l'objet d'« applications » extrapolées à partir du champ théorique. Elle ne relève pas non plus du seul domaine de l'intervention pratique (i.e. de l'enseignement). Elle constitue, pour la recherche, tout à la fois une porte d'entrée pour comprendre le fonctionnement des objets langagiers et un principe de formalisation de ces mêmes objets.

Dans le même esprit que ce qui vient d'être évoqué, le quatrième intérêt lié au projet de connaissance des didactiques réside en ce qu'elles peuvent prendre appui sur l'analyse des dispositifs de travail et/ou de remédiation ainsi que sur l'analyse de leurs effets pour penser les savoirs, les pratiques et leurs modélisations. En effet, ici encore, l'analyse des dispositifs et de leurs effets, que ces dispositifs aient été élaborés dans un cadre de recherche ou non - pourvu que cette analyse soit rigoureusement instrumentée (Reuter, dir., 2007, Ruellan, 2000) - peut revêtir des valeurs heuristiques fondamentales :

- en explorant les voies possibles de l'enseignement (Martinand, 1987), par exemple en ce qui concerne les modèles des savoirs, des pratiques, des écrits proposés aux apprenants ;

- en mettant au jour des modèles implicites des contenus, de l'enseignement, des apprenants... à l'œuvre dans ces dispositifs à l'insu de leur initiateur ;

- en éclairant les limites de tel ou tel modèle théorique sur le terrain de l'enseignement et des apprentissages...

Le dernier intérêt sur lequel je m'arrêterai ici tiens au fait que les didactiques constituent un espace de recherche privilégié pour mettre en œuvre la confrontation des modèles et des hypothèses des diverses disciplines de recherche lorsque celles-ci portent sur certaines dimensions de l'enseignement et des ap-

(19) Voir, par exemple, la difficulté à construire la partie appelée synthèse dans les dissertations lorsque les idées développées dans les parties appelées thèse et antithèse ne sont pas, en quelque sorte, exacerbées. 
prentissages. Elles constituent en effet un espace théorique privilégié dans la mesure où elles sont, structurellement, des espaces d'interaction (Reuter, 2005, 2007b). D'une part, parce qu'elles se situent à la croisée des disciplines de recherche qui traitent des contenus, de celles qui traitent de l'enseignement et de celles qui traitent des apprentissages ${ }^{(20)}$. D'autre part, parce qu'elles sont soumises à ce que j'ai appelé un horizon praxéologique, c'est-à-dire qu'elles tirent en grande partie leur existence et leur légitimité de difficultés d'enseignement et d'apprentissages existantes et qu'elles ont, en grande partie, le projet d'éclairer ces difficultés et de contribuer à leur résolution via les connaissances qu'elles produisent. C'est dans cette perspective, me semble-t-il, qu'elles sont amenées à explorer et à confronter des thèses qui, autrement, connaissent des existences séparées dans leur espace scientifique originel. J'ai ainsi pris, à diverses reprises (voir notamment Reuter, 2004b), l'exemple du rapport réflexif au langage avec d'un côté les thèses de la psychologie cognitive qui font de la dimension métalangagière une compétence technique nécessaire pour apprendre et, de l'autre, les thèses de certains sociologues (Lahire, 1993) qui appréhendent cette même dimension comme une disposition socialement construite participant de ce fait à la sélection socio-scolaire. Je pourrais, de la même façon, prendre l'exemple de la citation (Lidil, 2001) en montrant comment les didactiques peuvent permettre d'interroger les articulations entre des problèmes relevant selon certains théoriciens du domaine langagier, des problèmes relevant selon d'autres chercheurs du domaine de la cognition, et des problèmes attribuables selon d'autres théoriciens encore aux fonctionnements sociaux et à la biographie culturelle des sujets(biographie culturelle qui peut éclairer, au moins en partie, la manière dont les sujets se situent par rapport à des théoriciens « reconnus »).

\section{De quelques problèmes à débattre}

Il n'en demeure pas moins vrai que les didactiques ne constituent certainement pas - nul ne saurait d'ailleurs le prétendre - les seules disciplines aptes à traiter ces questions et que, de surcroit, elles se heurtent, elles aussi, à des problèmes spécifiques. Je n'évoquerai ici que cinq d'entre eux.

\subsection{La difficile collaboration entre disciplines de recherche}

La première de ces difficultés - et non la moindre !- consiste à penser les manières de pratiquer les collaborations entre disciplines de recherche, collaborations que chacun appelle certes de ses vœux mais qui demeurent généralement à l'état d'ébauche dans la mesure où cela se réduit, trop souvent encore, à une

(20) De surcroit, sur chacun de ces pôles, les didactiques sont amenées à mettre en interaction des domaines scientifiques différents (par exemple, sur le pôle des contenus, en matière d'écriture : les sciences du langage, les théories du texte, la psychologie cognitive, l'anthropologie, la sociologie,l'ethnologie...) 
juxtaposition d'interventions de différentes disciplines, s'ignorant les unes des autres au sein de telle ou telle recherche ou de tel ou tel colloque. Il me semble de surcroit que cela est d'autant plus difficile à penser qu'il n'existe pas encore - ou de manière très embryonnaire - de didactiques des disciplines universitaires ${ }^{(21)}$ qui, dans ce cas, pourraient tenter de se constituer en chevilles ouvrières de telles collaborations.

Il me semble encore que cette collaboration est d'autant plus difficile à mettre en œuvre que certaines représentations peuvent avoir la vie dure. Ainsi, par exemple, la didactique du français (ou de la langue maternelle) est souvent sollicitée par les autres disciplines sur la base d'ambiguïtés qui révèlent, ainsi que je l'évoquais dans un article précédent (Reuter, 2008), la difficulté des universitaires et des chercheurs à s'affranchir des cadres scolaires les plus classiques. On sollicite ainsi les didacticiens du français pour traiter des problèmes de lecture et d'écriture dans les différentes disciplines comme on sollicite le professeur de français dans le secondaire pour résoudre les problèmes de lecture et d'écriture en mathématiques, en sciences ou en histoire... comme si les modes de lecture et d'écriture et les difficultés qui leur sont liées n'étaient absolument en rien tributaires des disciplines concernées.

\subsection{Déterminer les configurations disciplinaires méthodologiquement pertinentes}

Une autre difficulté ${ }^{(22)}$ réside dans la détermination de la configuration disciplinaire pertinente pour analyser les genres, pratiques, difficultés des étudiants... en raison de la multiplicité des découpages institutionnels envisageables.

Ainsi, et pour ne prendre que l'exemple des Sciences de l'Éducation ${ }^{(23)}$, fautil, pour analyser les difficultés de lecture et d'écriture des étudiants, se situer par rapport aux Sciences de l'Éducation «en général 》 (en France et/ou dans d'autres pays ?), faut-il se situer par rapport à telle ou telle sous-discipline des Sciences de l'Éducation caractérisée soit par sa référence à telle ou telle autre discipline universitaire (psychologie de l'éducation ou sociologie de l'éducation, par exemple), soit par sa référence un champ de pratiques (travail social, éducation à la santé...) ou faut-il encore se situer par rapport à tel parcours de formation dans telle université (Sciences de l'Éducation à Genève, à Paris 5 ou à Lille 3) ?

(21) La question des relations entre didactiques des disciplines scolaires et didactiques des disciplines universitaires est, à mon sens, trop rarement posée et les réponses ne sont sans doute pas simples et variables selon les disciplines...

(22) Difficulté bien mise au jour par les recherches menées dans le cadre de l'Agence Nationale de la Recherche sous la direction d'Isabelle Delcambre et par les résultats fournis par B. Daunay, I. Delcambre et D. Lahanier-Reuter (voir, entre autres, Daunay et LahanierReuter, 2011 et Delcambre et Lahanier-Reuter, 2011).

(23) Mais cela me semble valable pour toute autre discipline. 


\subsection{Construire des catégories pertinentes quant aux sujets en difficulté et aux domaines problématiques}

La troisième famille de problèmes que rencontrent les didactiques réside dans la détermination des catégories d'étudiants en difficulté, dans l'affinement des catégories de difficultés, et dans le croisement de ces deux typologies.

Si on admet en effet, à la suite de nombre d'études empiriques, que les types de difficultés sont variables et que, de surcroit, les difficultés ne concernent pas les mêmes catégories d'étudiants, il parait nécessaire de dépasser ces constats trop généraux afin de tenter d'élaborer des cartographies les plus fines possibles des types de difficultés et des catégories d'étudiants concernés et afin d'étudier les croisements possibles de ces outils heuristiques. Ce qui - je me permets de le souligner - minimise dans l'enseignement supérieur, comme dans les autres ordres d'enseignement d'ailleurs, la place possible de manuels de conseils très généraux censés convenir à tous.

\subsection{Distinguer les types de littéracies auxquels on se réfère}

Il me parait encore indispensable - même si c'est sans doute une tâche très difficile - de distinguer ce qui peut relever de différents types de littéracie : l'ordre des littéracies potentiellement «communes », plus ou moins partagées, au sein d'une société donnée à un moment historique déterminé ; ce qui est du ressort des littéracies universitaires (1'université étant ici considérée comme une sphère socio-institutionnelle spécifique, sphère organisant donc des pratiques qui lui sont propres) ; et ce qui serait spécifique de littéracies disciplinaires. Cela afin de tenter de mieux analyser les relations et les interactions entre ces trois espaces. Cela afin aussi de tenter de mieux comprendre les causes possibles de difficultés et, conséquemment, de tenter d'élaborer des stratégies d'aide mieux articulées et/ou mieux ajustées...

\section{5. Évaluer les principes de modélisation des pratiques et des difficultés}

La dernière famille de problèmes sur laquelle je m'arrêterai concerne la détermination des principes de modélisation qui seraient les plus adéquats pour rendre compte des pratiques et des savoirs dans la perspective que j'ai tentée d'expliciter ici, c'est-à-dire en essayant de tenir compte des articulations possibles entre analyse des contenus, analyse des difficultés et analyse des stratégies de remédiation envisageables. Je proposerais volontiers ici de considérer - ainsi que je l'ai défendu ailleurs - que, chacun pour des raisons différentes, les modèles fonctionnant sur les notions d'unité ou d'homogénéité (par exemple, les typologies textuelles), d'algorithme (par exemple, certaines modélisations des opérations cognitives censées être à l'œuvre dans l'écriture) ou d'application de règles (notion en l'occurrence à préciser), ne me paraissent pas satisfaisants, ne serait-ce qu'en fonction de leur trop grand degré de généralité et de leur absence de prise en compte des pratiques et des représentations des ac- 
teurs. Ce qui m'a amené de plus en plus (Reuter, 2004a) à explorer des modèles raisonnant en termes de résolution plus ou moins (in)consciente de problèmes mal définis aux solutions multiples, et/ou de gestion de dimensions hétérogènes et/ou de tensions (par exemple entre position de formé et position de chercheur dans l'écriture de recherche en formation : mémoires, thèses...).

Dès lors, il s'avère nécessaire d'abandonner le terrain des certitudes, des modèles sans aspérités et des prescriptions assurées pour aborder les espaces, moins défrichés mais sans nul doute plus stimulants, des incertitudes, du flou, des équilibres instables et des ajustements tâtonnants.

J'ai bien conscience, au moment de conclure cet article, d'avoir finalement, en tentant d'expliciter les intérêts de la perspective didactique en matière de littéracies, soulevé bien plus de questions que je n'ai résolu de problèmes. Mais c'est peut-être là le lot de toute démarche de recherche. C'est peut-être aussi une nécessité lorsque se développe véritablement un nouveau chantier de recherches. J'espère, en tout cas, au-delà de l'éclairage que j'aurais pu apporter sur les didactiques, notamment quant à leur capacité à traiter certaines des questions afférentes aux littéracies universitaires, avoir ouvert des pistes susceptibles d'aider à penser les points communs et les différences avec d'autres disciplines de recherche se développant parallèlement dans les monde francophone ou anglo-saxon, pistes susceptibles aussi de favoriser des collaborations entre disciplines de recherche sur ces questions. 


\section{Références}

Astolfi, J.-P., Darot, E., Ginsburger-Vogel, Y., Toussaint, J. (1997) : Mots-clés de la didactique des sciences, Bruxelles, De Boeck.

Astolfi, J.-P., DeVElay, M. (1989) : La didactique des sciences, Paris, P.U.F.

BARRÉ DE MiniAC, C., Brissaud, C., Rispail, M. (2004) : La littéracie. Conceptions théoriques et pratiques d'enseignement de la lecture-écriture, $\mathrm{Pa}$ ris, L'Harmattan.

BorzeiX, A., FraencKel, B. (2001) : Langage et travail. Communication, cognition, action, Paris, CNRS éditions.

Bourdieu, P. (1984) : Homo academicus, Paris, Les éditions de Minuit.

Bourdieu, P., CHARTIER, R. (2010) : Le sociologue et l'historien, Paris, Éditions Agone, en collaboration avec Raisons d'Agir et Ina Éditions.

Charlot, B., B Autier, E., RocheX, J.-Y. (1992) : École et savoirs dans les banlieues et ailleurs, Paris, Armand Colin.

CHEVAllard, Y. (1985/1991) : La transposition didactique. Du savoir savant au savoir enseigné, Grenoble, La Pensée Sauvage.

COHEN-AzRIA, C. (2007) : «Niveaux de formulation », dans Reuter Yves, dir. : Dictionnaire des concepts fondamentaux des didactiques, Bruxelles, De Boeck, 145-147.

DAUnAY, B. (2007) : « Système didactique-Triangle didactique », dans Reuter Yves, dir., : Dictionnaire des concepts fondamentaux des didactiques, Bruxelles, De Boeck, 207-213.

DAUnAY, B., LAHANIER-REUTER, D. (2011) : «Les genres d'écrits dans la formation supérieure : étude comparative en formation professionnelle d'enseignants et formation universitaire générale », Bulletin suisse de linguistique appliquée, Neuchâtel, Université de Neuchâtel,101-113.

DelCAMBRE, I. (2007a) : «Genres du discours », dans Reuter Yves, dir. : Dictionnaire des concepts fondamentaux des didactiques, Bruxelles, De Boeck, 117-122.

- (2007b) : "Métalangage - activité métalinguistique », dans Reuter Yves, dir. : Dictionnaire des concepts fondamentaux des didactiques, Bruxelles, De Boeck, 127-133.

DelCAmbre, I., LAHANIER-REUTER, D. (2011) : «Continuités et ruptures dans les pratiques d'écriture à l'université : l'exemple de cursus en Sciences Humaines », Bulletin suisse de linguistique appliquée, Neuchâtel, Université de Neuchâtel,115-130.

DELFORCE, B. (1985) : "Approches didactiques de la production d'un écrit "fonctionnel" : les difficultés de la dissertation ", Pratiques, n 48, Les écrits non fictionnels, Metz, CRESEF, décembre, 35-52.

- (1986) : «Les élèves face aux devoirs et aux copies corrigées : bavardages inutiles, réactions aberrantes ou discours révélateurs », Bulletin du CERTEIC, $\mathrm{n}^{\circ} 7$, Évaluer les écrits : correction des copies, production de 
textes, démarches d'apprentissage, Villeneuve d'Ascq, Université de Lille 3, 91-116.

Giguère, J., Reuter, Y. (2004) : «Présentation d'une grille d'analyse de l'univers de l'écrit à l'école primaire ", Cahiers Théodile, ${ }^{\circ} 4$, Université de Lille 3, janvier, 103-122.

Goody, J. (1977/1979) : La raison graphique. La domestication de la pensée sauvage, édition française, Paris, Les éditions de Minuit.

LAhANiER-Reuter, D., Reuter, Y. (2002) : « Écrits et apprentissages. Première approche dans quatre disciplines au collège ", Pratiques, $\mathrm{n}^{\circ} 113$ 114, Images du scripteur et rapports à l'écriture, Metz, CRESEF, juin, 728.

LAHIRE, B. (1993) : Culture écrite et inégalités scolaires. Sociologie de l'" échec scolaire " à l'école primaire, Lyon, Presses Universitaires de Lyon.

- (2008) : "La forme scolaire dans tous ses états », Revue suisse des Sciences de l'Éducation, n³0, Gros plan sur la forme scolaire, 229-256.

LIDIL (2001) : Apprendre à citer le discours d'autrui, $\mathrm{n}^{\circ} 24$, Université Stendhal, Grenoble III, Lidilem, décembre (Boch Françoise, Grossmann Francis, dir.).

- (2003) : La littéracie. Vers de nouvelles pistes de recherche didactique, Université Stendhal, Grenoble, $\mathrm{n}^{\circ} 27$, juillet.

MARTINAND, J.-L. (1987) : «Quelques remarques sur les didactiques des disciplines », Les Sciences de l'Éducation pour l'ère nouvelle, $\mathrm{n}^{\circ} 1-2$, Didactiques et Sciences de l' 'Education, 23-29.

REUTER, Y. (2003) : «La représentation de la discipline ou la conscience disciplinaire », La Lettre de la DFLM, n 32, AIDFLM, Namur, 18-22.

- (1996) : Enseigner et apprendre à écrire. Construire une didactique de l'écriture, Paris, E.S.F.

- (2004a) : «Analyser les problèmes de l'écriture de recherche en formation ", Pratiques, $\mathrm{n}^{\circ}$ 121-122, Normes et pratiques de l'écrit dans le supérieur, Metz, CRESEF, juin, 9-27.

- (2004b) : "Quelques spécificités des approches didactiques de l'écriture ", Linx, $\mathrm{n}^{\circ} 51$, Théories de l'écriture et pratiques scolaires, Paris X, 41-54.

- (2005) : «Didactique du français : éléments de réflexion et de proposition », dans Chiss Jean-Louis, David Jacques, Reuter Yves, dir. : Didactique du français. Fondement d'une discipline, Bruxelles, De Boeck, 211231.

— (2007a) : «Disciplines scolaires », dans Reuter Yves, dir. : Dictionnaire des concepts fondamentaux des didactiques, Bruxelles, De Boeck, 85-89.

— (2007b) : «Didactiques », dans Reuter Yves, dir. : Dictionnaire des concepts fondamentaux des didactiques, Bruxelles, De Boeck, 69-73.

- (2007c) : « La conscience disciplinaire. Présentation d'un concept », Education et Didactique, volume 1, ${ }^{\circ} 2$, Presses Universitaires de Rennes, Septembre, 54-70. 
- (2008) : «Interroger l'appareillage conceptuel de la didactique du français ", La Lettre de l'AIRDF, ${ }^{\circ}$ 42, La didactique du français : coup d'œil vers le passé. En hommage à Jean-François Halté, juin, 18-21.

- ed. (2007) : Une école Freinet. Fonctionnement et effets d'une pédagogie alternative en milieu populaire, Paris, l'Harmattan.

REUTER, Y., LAHANIER-REUTER, D. (2007) : «L'analyse des disciplines : quelques problèmes pour la recherche en didactique », dans Falardeau Érick, Fisher Carole, Simard Claude, Sorin Noëlle, eds. : La didactique du français. Les voies actuelles de la recherche, Québec, les presses de l'université Laval, 27-42.

- (2007) : « Analyzing writing in academic disciplines : a few concepts », L1 Educational Studies in Language and Literatie, 8(2), Kluwer, Academic Publishers, Amsterdam, 47-57.

RUELlan, F. (2000) : Un mode de travail didactique pour l'enseignement-apprentissage de l'écriture au cycle 3 de l'école primaire, Thèse de doctorat, Université de Lille 3, Atelier National de Reproduction des Thèses de Lille.

VINCENT, G. (1980) : L'école primaire française, Lyon, Presses Universitaires de Lyon.

- (1994). L'éducation prisonnière de la forme scolaire? Scolarisation et socialisation dans les sociétés industrielles, Lyon, Presses Universitaires de Lyon. 